\title{
Cepa nativa de Beauveria pseudobassiana como agente potencial en el control biológico de Monochamus galloprovincialis, vector del nematodo de la madera del pino
}

\author{
Álvarez-Baz, G. ${ }^{*}$, Fernández-Bravo, M. ${ }^{2}$, Pajares, J. ${ }^{1}$, Quesada-Moraga, E. ${ }^{2}$ \\ ${ }^{1}$ Instituto de Investigación en Gestión Forestal Sostenible, Universidad de Valladolid. \\ Campus de La Yutera. Avda. de Madrid, 44. 34004, Palencia. \\ ${ }^{2}$ Departamento de ciencias agrícolas y forestales, ETSIAM, Universidad de Córdoba. \\ Campus de Rabanales. Edificio C4 Celestino Mutis. 14071 Córdoba. \\ *Autor para correspondencia: gonzalbaz@gmail.com
}

\section{Resumen}

Se aislaron tres cepas de hongos entomopatógenos en ejemplares adultos de Monochamus galloprovincialis (Olivier) (Coleptera: Cerambycidae) infectados de forma natural que resultaron pertenecer a las especies Isaria farinosa (Holmsk.) Fr., Lecanicillium attenuatum (Zare \& W. Games) y Beauveria pseudobassiana (Bals.) Vuill. Esta es la primera vez que se han aislado estos hongos en ejemplares de M. galloprovincialis. En nuestros ensayos, la cepa de B. pseudobassiana (EABps 11/01-Mg) resultó ser la más virulenta para este insecto. Se investigó su capacidad de transmisión horizontal y vertical en formulación acuosa y seca. La formulación acuosa no mostró evidencias de ningún tipo de transmisión. Sin embargo, todos los insectos infectados de forma horizontal con la formulación seca murieron y su tiempo medio de vida se redujo significativamente. El número medio de mordeduras de oviposición realizadas, huevos puestos, larvas vivas 5 días tras la puesta y orificios de entrada para pupar en el xilema 6 meses después fue significativamente inferior tanto en hembras expuestas directamente al hongo como en aquellas que lo recibieron por transmisión horizontal en comparación con las hembras control, mostrando que una reducción de la progenie inducida de forma horizontal es posible. Estos resultados manifiestan el potencial de la cepa aislada de Beauveria pseudobassia$n a$ como agente regulador de la población de este insecto. A través de técnicas de autodiseminación, esta cepa podría ser usada en el control integrado del vector del nematodo de la madera del pino como una nueva herramienta para el manejo de la enfermedad del marchitamiento de los pinos.

Palabras clave: hongos entomopatógenos, enfermedad del marchitamiento de los pinos, manejo integrado, Bursaphelenchus xylophilus, Isaria farinosa, Lecanicillium attenuatum. 


\section{Introducción}

El nematodo de la madera del pino, Bursaphelenchus xylophilus (Steiner and Buhrer) Nickle (Nematoda; Aphelenchoididae), es uno de los organismos introducidos más dañinos del último siglo. Aunque se considera un patógeno de debilidad en su Norteamérica nativa, causa un decaimiento y rápida muerte en pinos y otras coníferas (Wingfield, 1982). Después de haberse extendido por el sureste asiático durante décadas causando graves pérdidas económicas y ambientales (Shin, 2008; Zhao, 2008) se registró por primera vez en portugal en 1999 (Mota et al., 1999), por donde se extendió devastando grandes masas (Daub, 2008; Rodrigues, 2008). Finalmente cruzó a España en 2008 (Espárrago, 2012), donde se han declarado cuatro focos que se están erradicando.

Este nematodo necesita de insectos vectores del género Monochamus (Coleoptera: Cerambycidae) para infectar nuevos árboles hospedantes (Linit, 1988; Linit y Akbulut, 2008). Son insectos que colonizan árboles gravemente dañados por fuego, sequías u otros organismos. Los ejemplares adultos se alimentan de ramillos jóvenes de árboles sanos, al principio para su maduración sexual y después como alimentación de mantenimiento. Las hembras fecundadas realizan mordeduras en la corteza de árboles dañados en el interior de las cuales depositan sus huevos. Los árboles sanos son infectados con nematodos cuando los adultos se alimentan de ramillos (Mamiya and Enda, 1972) pero el nematodo también se puede transmitir durante la oviposición (Evans et al., 2008). Hasta ahora M. galloprovincialis (Olivier) es el único vector del nematodo en Europa (Sousa et al., 2001). Coloniza pinos, píceas y abetos (Vives, 2000) y está distribuido por el continente europeo y norte de África. El control de nematodo ha sido difícil en todos los países (Kamata, 2008) y su manejo directo es inviable en masas forestales, siendo el control de los insectos vectores la mejor alternativa. En algunos países se han empleado insecticidas químicos (Shin, 2008; Zhao et al., 2008) pero se consideran desaconsejables debido a su eficacia cuestionable y el peligro potencial para la salud humana y medioambiental.

Se han realizado intentos de control biológico de Monochamus en Asia (Shimazu, 2008) y se consiguieron buenos niveles de parasitismo por medio de Dastarcus helophoroides (Coleoptera: Bothrideridae) y Scleroderma guani (Hymenoptera: Bethylidae) en M. alternatus en China, especialmente cuando los parasitoides actuaban en presencia de Beauveria bassiana (Shimazu, 2008; Xu, 2008). También se han obtenido buenos resultados con los nematodos entomopatógenos Contortylenchus genitalicola y Steinernema carpocapsae pero su aplicación práctica no ha sido desarrollada (Phan, 2008; Shimazu, 2008). Los hongos entomopatógenos que infectan a través de la cutícula tienen un gran potencial como agentes de control biológico. Además de matar a los insectos, los hongos entomopatógenos han demostrado efectos subletales en la reproducción (Quesada-Moraga et al., 2004; 2008). Algunas especies de hongos entomopatógenos aisladas de sus hospedantes han sido utilizadas como agentes de control en otros países (Shin et al., 2011). Se han aislado varias cepas de Beauveria bassiana muy virulentas en poblaciones de M. alternatus en Asia (Shimazu, 2008; Shin et al., 2009), sin embargo su aplicación práctica en con- 
trol biológico de insectos es limitada debido a sus estadios larvarios ocultos (Kishi, 1995). Se han probado diferentes métodos de liberación de hongos entomopatógenos como la pulverización, la aplicación manual subcortical o la dispersión mediante insectos (Shimazu et al., 1995; Shimazu y Sato, 2003; Shimazu, 2008), aunque ninguno de ellos se ha mostrado lo suficientemente eficaz. Shimazu et al. (1995) desarrolló un sistema de aplicación de B. bassiana a M. alternatus mediante el uso de fibras textiles eficaz sobre adultos (Okitsu, 2000; Shimazu, 2004a) y larvas (Shimazu et al., 1995; Shimazu y Sato, 2003). En Europa se han probado cuatro aislados del suelo de $B$. bassiana y una formulación comercial sobre M. galloprovincialis con buenos resultados (Francardi et al., 2003). Naves (2008) encontró que $B$. bassiana era el factor más importante en la muerte natural de M. galloprovincialis en el área de Portugal afectada por el nematodo. Esto sugiere que en poblaciones europeas de $M$. galloprovincialis se pueden aislar cepas nativas de B. bassiana suficientemente virulentas como para infectar a esta especie.

El reciente avance en el conocimiento de la ecología química de M. galloprovincialis (Ibeas et al., 2008, 2007; Pajares et al., 2004) y desarrollo de cebos y trampas altamente eficaces (Pajares, 2010, Álvarez et al., 2014) abren una oportunidad para el uso de los hongos entomopatógenos en el manejo de esta enfermedad. Las poblaciones de insectos podrían ser inoculadas con cepas de $B$. bassiana mediante técnicas de "atrae e infecta" y dispositivos de autodiseminación (Francardi et al., 2013; Klein and Lacey, 1999; Yasuda, 1999). Los insectos inoculados en estos dispositivos deberían ser capaces de transmitirlo a otros insectos adultos durante la cópula (transmisión horizontal) o a su progenie (transmisión vertical). En este trabajo se presentan resultados de cuatro años de investigación en el aislamiento de cepas nativas de hongos entomopatógenos de $M$. galloprovincialis en España.

\section{Material y métodos}

\subsection{Insectos}

Para obtener adultos de M. galloprovincialis para los ensayos se recogieron trozas de árboles quemados en incendios naturalmente colonizadas por este insecto y trozas cebadas. Para ello se colocaron 120 trozas de Pinus sylvestris de 120 x 8-15 $\mathrm{cm}$ cebadas con Galloprotect 2D (SEDQ, Barcelona) en varios grupos a lo largo de cortafuegos en Tabuyo del Monte (León, 42 ${ }^{\circ} 18^{\prime} 13.2^{\prime \prime} \mathrm{N}, 6^{\circ} 11^{\prime} 27.6^{\prime \prime} \mathrm{W}$ ) durante los veranos de 2011 y 2012. Las trozas mejor colonizadas se recogieron al final del verano y se mantuvieron en una jaula exterior hasta la emergencia de los adultos al año siguiente. Los adultos emergentes se mantuvieron individualmente en tarros de vidrio de 1 litro bajo fotoperiodo y temperatura de $15: 9$ h y $22: 15^{\circ} \mathrm{C}$ (luz:oscuridad), alimentados semanalmente con ramillos frescos de pino.

Las larvas utilizadas en los ensayos se obtuvieron de cría controlada en laboratorio, para lo cual hembras de M. galloprovincialis realizaron la puesta en trozas de P. sylvestris recolectadas en Saldaña (Palencia, $42^{\circ} 37^{\prime} 40.1^{\prime \prime} \mathrm{N}, 4^{\circ} 47^{\prime} 30.0^{\prime \prime} \mathrm{W}$ ). Las 
trozas fueron posteriormente colocadas en cajas de plástico $(60 \times 30 \times 30 \mathrm{~cm})$ donde las larvas se desarrollaron para ser recolectadas varios días después.

\subsection{Aislamiento y caracterización de los hongos}

En Febrero de 2010 se recolectaron 240 trozas colonizadas por M. galloprovincialis de árboles incendiados en Cuevas del Valle (Ávila, $40^{\circ} 17^{\prime} 58.3^{\prime}$ ' N, $5^{\circ} 01$ ' 17.2 W). Tras la emergencia de los adultos se seleccionaron las 32 que menor número de adultos emergentes habían proporcionado y fueron cortadas en láminas de $1 \mathrm{~cm}$ de grosor con una sierra de cinta. De estas trozas se recolectaron 45 muestras de larvas, pupas y adultos muertos, así como pedazos de madera con micelio en las galerías de los insectos. Del mismo modo en 2011 se obtuvieron 39 muestras de un total de 250 trozas recogidas en el mismo campo. Los insectos muertos fueron superficialmente esterilizados con hipoclorito de sodio (1\%) durante un minuto y lavados en agua esterilizada durante un minuto. Posteriormente se colocaron sobre papel de filtro estéril en placas petri selladas con Parafilm durante dos semanas a $25^{\circ} \mathrm{C}$ en oscuridad para facilitar el desarrollo fúngico. Los hongos fueron aislados directamente de los insectos adultos y pupas que presentaban crecimiento micelial usando agar sabouraud de glucosa-cloranfenicol, en el que fueron cultivados durante dos semanas a $25^{\circ} \mathrm{C}$ y oscuridad para su posterior identificación morfológica y molecular. Los hongos aislados se clasificaron a nivel de género mediante microscopía óptica (X400) con clave taxonómica (Barnet and Hunter, 2006). Para la caracterización molecular se inocularon placas de extracto de malta-agar cubiertas con cefolán estéril, del que posteriormente se obtuvo por raspado el material biológico del que se extraería ADN micelial (Raeder and Broda, 1985).

El gen nuclear EF-1 $\alpha$ fue amplificado, secuenciado y analizado en las diferentes muestras. Un fragmento de 1100 pares de bases de dicho gen se amplificó con los primers tef1 fw (O’Donnell et al., 1998) y 1750-R (Garrido-Jurado et al., 2011). Las alineaciones se generaron utilizando el programa MegAlign (DNASTAR, 198992, Londres, Reino Unido). Todos los aislados de hongos entomopatógenos obtenidos fueron depositados en la colección de cultivos del Departamento de Ciencias Agrarias y Forestales, ETSIAM, Universidad de Córdoba, España.

\subsection{Ensayo de patogenicidad inicial de los hongos aislados sobre larvas y adultos de M. galloprovincialis}

Se realizaron dos bioensayos para determinar la patogenicidad de los tres hongos aislados sobre adultos y larvas de $M$. galloprovincialis. Para cada aislado se realizó una suspension acuosa de conidios en agua estéril destilada ( $0.1 \%$ Tween 80$)$ ajustada a $1 \times 10^{8}$ conidios $/ \mathrm{ml}$. Los insectos adultos de 2-3 semanas de vida para asegurar su madurez sexual (Tomiczek and Hoyer-Tomiczek, 2008) fueron introducidos individualmente en una cestilla metálica y sumergidos en la suspensión conidial durante 20 segundos. Del mismo modo los insectos control fueron sumergidos en agua destilada con $0.1 \%$ de Tween 80 . Tras la inmersión, se mantuvieron in- 
dividualmente en tarros de vidrio de 1 litro bajo fotoperiodo y temperatura de 15:9 h y $22: 15^{\circ} \mathrm{C}$ (luz:oscuridad), alimentados semanalmente. Se hizo un seguimiento de su mortalidad durante 30 días y los insectos muertos fueron superficialmente esterilizados con hipoclorito sódico (1\%) durante un minuto. Posteriormente fueron colocados en placas petri con papel de filtro estéril, selladas con Parafilm y mantenidas a $25^{\circ} \mathrm{C}$ en la oscuridad para facilitar el desarrollo micelial sobre los cuerpos. Siguiendo el mismo procedimiento se trataron cinco larvas. Tras la inmersión las larvas que habían recibido el mismo tratamiento fueron introducidas en una troza de $P$. sylvestris $(8-15 \times 50 \mathrm{~cm})$ a través de orificios practicados en la corteza. Se vigiló la actividad larvaria durante los días siguientes mediante el serrín generado, y cuando ésta se detuvo la galería fue descortezada en busca de la larva, que fue recolectada siguiendo el mismo procedimiento que con los adultos para favorecer el desarrollo micelial.

\subsection{Ensayos de virulencia de B. pseudobassiana sobre adultos de M. galloprovincialis}

Para el ensayo de virulencia se prepararon cuatro suspensiones conidiales $\left(1 \times 10^{5}\right.$, $1 \times 10^{6}, 1 \times 10^{7}$ y $1 \times 10^{8}$ conidios $/ \mathrm{ml}$ ) de $B$. pseudobassiana. Del modo anteriormente descrito se sumergieron 18 adultos durante 20 segundos mientras que los 18 insectos control fueron sumergidos en agua destilada estéril con $0.1 \%$ Tween 80 . Tras la inmersión, los insectos se mantuvieron individualizados en tarros de vidrio de 1 litro bajo las condiciones anteriormente descritas. Se hizo un seguimiento de su mortalidad durante 30 días y los insectos muertos fueron procesados para favorecer el desarrollo micelial del modo anteriormente descrito.

\subsection{Transmisión horizontal de B. pseudobassiana entre adultos de $M$. galloprovincialis}

\subsubsection{Utilizando suspensión conidial}

Ejemplares adultos y maduros de $M$. galloprovincialis fueron inoculados por inmersión en una suspensión conidial de B. pseudobassiana $\left(1 \times 10^{8}\right.$ conidios $\left./ \mathrm{ml}\right)$ o en agua destilada estéril con $0.1 \%$ Tween 80 . Posteriormente los insectos se emparejaron en 10 parejas de cada una de las siguientes combinaciones:

- Machos control con hembras control.

- Machos inoculados con hembras control.

- Machos control con hembras inoculadas.

- Machos inoculados con hembras inoculadas.

Cada pareja se introdujo en una caja de plástico con dos trozas de P. sylvestris $(15 \times 50 \mathrm{~cm})$ para realizar la cópula y oviposición. Cada dos días se contaron las mordeduras de oviposición sobre las trozas y la pareja de insectos se retiró cuando habían realizado al menos 15 mordeduras. Los insectos retirados fueron individualizados en tarros de vidrio y mantenidos de la forma anteriormente descrita. 
Se registró su mortalidad durante los 30 días siguientes y los insectos muertos fueron procesados para favorecer el desarrollo micelial del modo anteriormente descrito.

\subsubsection{Utilizando formulación seca}

Se realizó un ensayo similar en el que los insectos maduros fueron inoculados utilizando una formulación seca en talco ajustada a $4.25 \times 10^{9}$ conidios/g de B. pseudobassiana. Para la inoculación los insectos fueron forzados a caminar sobre la preparación durante 20 segundos asegurando el completo recubrimiento del cuerpo. Los insectos control fueron obligados a caminar sobre polvo de talco puro. Posteriormente los insectos fueron emparejados en 10 parejas para cada una de las cuatro combinaciones descritas anteriormente. Cada pareja se introdujo en una caja de plástico con una troza de $P$. sylvestris $(10 \times 30 \mathrm{~cm})$ para realizar la cópula y oviposición. Para evitar la sobresaturación de la troza con larvas, ésta fue reemplazada por otra cuando la hembra había realizado 5 mordeduras de puesta. Cada pareja realizó al menos 20 mordeduras de puesta, tras lo cual los insectos fueron individualizados en tarros de vidrio en las condiciones anteriormente descritas. Se registró su mortalidad cada dos días durante los 30 días siguientes y los insectos muertos fueron procesados para favorecer el desarrollo micelial del modo anteriormente descrito.

\subsection{Transmisión vertical de B. pseudobassiana entre adultos de $M$. galloprovincialis}

\subsubsection{Utilizando suspensión conidial}

Dos semanas después de que los insectos realizaran la oviposición en el experimento de transmisión horizontal descrito en el apartado 2.5.1, una de las trozas fue cuidadosamente descortezada bajo las mordeduras de puesta en busca de larvas neonatas, huevos muertos y mordeduras vacías (en las que no se llegó a realizar la puesta). Del mismo modo, dos semanas después (cuatro después de la oviposición) se inspeccionó la segunda troza en busca de progenie. En ambos casos los huevos y larvas muertos encontrados fueron procesados para favorecer el desarrollo micelial del modo anteriormente descrito.

\subsubsection{Utilizando formulación seca}

Cinco días después de que los insectos realizaran la oviposición en el experimento de transmisión horizontal descrito en el apartado 2.5.2, las mordeduras de puesta fueron examinadas de forma no destructiva en busca de actividad larvaria. Las mordeduras que no mostraron actividad fueron descortezadas para corroborar la presencia de progenie y los huevos o larvas muertos encontrados fueron recogidos. Las larvas activas fueron registradas y se les permitió completar su desarrollo para entrar a pupar en el xilema. Seis meses después, las trozas fueron descortezadas para contar el número de entradas al xilema. El número de huevos puestos se calculó como los huevos muertos más el número de larvas vivas y muertas encontradas. 
Tanto los huevos como las larvas muertas encontrados fueron procesados para favorecer el desarrollo micelial del modo anteriormente descrito.

\subsection{Análisis estadístico}

Los datos de mortalidad se analizaron con un modelo lineal generalizado (distribución binomial; link=logit). Las comparaciones entre tratamientos se realizaron mediante test $\chi^{2}$ (JMP 8.0, 2008 SAS Institute Inc.). El porcentaje de mortalidad causado por $B$. pseudobassiana en los ensayos de transmisión horizontal se corrigió para la mortalidad de los controles utilizando la fórmula de Abbot (Abbott, 1925). Se utilizó el análisis de supervivencia de Kaplan-Meier para determinar la mortalidad acumulada durante el periodo de seguimiento (Kaplan et al., 1958). Los valores de concentración media letal $\left(\mathrm{LC}_{50}\right)$ se estimaron mediante análisis probit (Finney, 1971). El tiempo estimado para la muerte del 50\% de los insectos se determinó mediante análisis probit para datos correlacionados (Throne et al., 1995). Para estos análisis se utilizó el paquete de software SPSS 15.0 para Windows (SPSS Inc. 2002).

En los experimentos de transmisión vertical, las mordeduras de puesta, huevos puestos, larvas vivas y muertas y entradas al xilema se analizaron ajustando modelos lineales generalizados con una distribución de errores de tipo Poisson o quasiPoisson (en caso de sobredispersión) (Crawley, 2007). Las comparaciones de medias se llevaron a cabo mediante el test de diferencia significativa honesta de Tukey. Estos análisis se realizaron con el paquete de software R 2.11.1 (R Core Team, 2012).

\section{Resultados}

\subsection{Aislamiento y catacterización de hongos entomopatógenos}

Se obtuvieron tres aislados de cadáveres de $M$. galloprovincialis. La observación óptica permitió identificar los géneros Beauveria, Isaria y Lecanicillium. El análisis molecular y comparación con GenBank (Tab. 1), permitieron identificar las especies Beauveria pseudobassiana, Isaria farinosa y Lecanicillium attenuatum.

\subsection{Patogenicidad inicial de los hongos aislados sobre larvas y adultos de M. galloprovincialis}

Sólo Beauveria pseudobassiana resultó ser altamente patógena sobre los adultos y larvas de M. galloprovincialis $\left(\chi^{2}(3)=23.61, \mathrm{p}<0.001 ; \chi^{2}(3)=17.54, \mathrm{p}<0.001\right.$, respectivamente) causando $100 \%$ de mortalidad en ambos casos. Del mismo modo el tiempo medio de vida (AST) de adultos y larvas tratados con B. pseudobassiana fueron significativamente menores (13.30 y 8.60 días respectivamente) en comparación con Isaria farinosa y Lecanicillium attenuatum (Tab. 2). 
Tabla 1. Cepas y número de GenBank para las secuencias de referencia utilizadas en el análisis de filogenia.

\begin{tabular}{|c|c|c|}
\hline Cepa & Especie & GenBank \\
\hline ARSEF 2181 & Purpureocillium lilacinum & EF468790 \\
\hline CBS 431.87 & “ & EF468791 \\
\hline ARSEF 7487 & Metarhizium anisopliae & DQ463996 \\
\hline ARSEF 7450 & “ & EU248852 \\
\hline ARSEF 727 & M. robertsii & DQ463994 \\
\hline ARSEF 4739 & “ & EU248848 \\
\hline ARSEF 4179 & M. brunneum & EU248854 \\
\hline ARSEF 4152 & “ & EU248853 \\
\hline ARSEF 2107 & “ & EU248855 \\
\hline ARSEF 842 & Beauveria bassiana & AY531963 \\
\hline ARSEF 1479 & “ & AY531891 \\
\hline ARSEF 753 & “ & AY531955 \\
\hline ARSEF 843 & “ & AY531964 \\
\hline ARSEF 2694 & B. varroae & HQ881004 \\
\hline ARSEF 8257 & “ & HQ881002 \\
\hline ARSEF 985 & B. brogniartii & HQ880978 \\
\hline ARSEF 10277 & “ & HQ880989 \\
\hline ARSEF 5689 & B. sungii & AY531939 \\
\hline ARSEF 1689 & “ & AY531899 \\
\hline ARSEF 4933 & B. pseudobassiana & AY531938 \\
\hline ARSEF 3405 & “ & AY531931 \\
\hline Jch041 & Lecanicillium attenuatum & KC633274 \\
\hline CBS402.78 & “ & EF468782 \\
\hline OSC111006 & Isaria farinosa & EF469065 \\
\hline OSC111055 & “ & DQ522348 \\
\hline ARSEF 5050 & Cordyceps militaris & HQ881020 \\
\hline
\end{tabular}

\subsection{Virulencia de B. pseudobassiana sobre adultos de M. galloprovincialis}

La mortalidad media a los 30 días de la inoculación fue del 44.4\% al 100\% para las concentraciones de $1 \cdot 10^{5}$ a $1 \cdot 10^{8}$ mientras que la del control fue del $16.6 \%$. El análisis probit dio un coeficiente de $0.70 \pm 0.18$ (pendiente $\pm \mathrm{ES}$ ) y el valor de $\chi^{2}$ no resultó significativo $\left(\chi^{2}=1.04,2 \mathrm{df}\right)$ con un valor $\mathrm{LC}_{50} \mathrm{de} 2.05 \times 10^{5}$ conidios $/ \mathrm{ml}$. El análisis de mortalidad acumulada produjo un resultado de (pendiente $\pm \mathrm{SE}=3.23 \pm 1.62 \chi^{2}=0.72 ; 4$ df $\mathrm{y} \mathrm{LT}_{50}=15.03$ y pendiente $\pm \mathrm{SE}=4.51 \pm 0.84, \chi^{2}=0.88,2$ df y $\mathrm{LT}_{50}=5.07$ ) para las concentraciones de $1 \times 10^{7}$ y $1 \times 10^{8}$ conidios $/ \mathrm{ml}$ respectivamente. 
Tabla 2. Patogenicidad de los hongos entomopatógenos contra adultos y larvas de Monochamus galloprovincialis.

\begin{tabular}{|c|c|c|c|c|c|}
\hline \multirow{3}{*}{$\begin{array}{c}\text { Especies } \\
\text { de hongos }\end{array}$} & \multicolumn{5}{|c|}{ Patogenicidad contra adultos } \\
\hline & \multicolumn{2}{|r|}{ Mortalidad (\%)b } & \multicolumn{3}{|c|}{ Análisis de supervivencia Kaplan-Meier } \\
\hline & Total & Crecimiento micelial & $\begin{array}{c}\mathrm{ASTa} \\
(\mathrm{media} \pm \mathrm{SE})\end{array}$ & $\begin{array}{l}\text { Inte } \\
\text { conf }\end{array}$ & $\begin{array}{l}\mathrm{de} \\
95 \%\end{array}$ \\
\hline \multirow{5}{*}{$\begin{array}{l}\text { Control } \\
\text { Beauveria pseudobassiana } \\
\text { Lecanicillium attenuatum } \\
\text { Isaria farinosa }\end{array}$} & & & & $\operatorname{Inf}$ & Sup \\
\hline & $30 \mathrm{a}$ & $0 \mathrm{a}$ & $26.90 \pm 1.83 \mathrm{a}$ & 23.30 & 30.49 \\
\hline & $100 \mathrm{~b}$ & $80 \mathrm{~b}$ & $13.30 \pm 2.49 b$ & 8.40 & 18.19 \\
\hline & $30 \mathrm{a}$ & $20 \mathrm{a}$ & $26.50 \pm 2.18 \mathrm{a}$ & 22.21 & 30.78 \\
\hline & $10 \mathrm{a}$ & $0 \mathrm{a}$ & $29.80 \pm 0.19 \mathrm{a}$ & 29.42 & 30.17 \\
\hline \multirow{3}{*}{$\begin{array}{c}\text { Especies } \\
\text { de hongos }\end{array}$} & \multicolumn{5}{|c|}{ Patogenicidad contra larvas } \\
\hline & \multicolumn{2}{|r|}{ Mortalidad (\%)b } & \multicolumn{3}{|c|}{ Análisis de supervivencia Kaplan-Meier } \\
\hline & Total & Crecimiento micelial & $\begin{array}{c}\mathrm{ASTa} \\
(\text { media } \pm \text { SE })\end{array}$ & $\begin{array}{l}\text { Inte } \\
\text { conf }\end{array}$ & $\begin{array}{l}\mathrm{de} \\
95 \%\end{array}$ \\
\hline \multirow{5}{*}{$\begin{array}{l}\text { Control } \\
\text { Beauveria pseudobassiana } \\
\text { Lecanicillium attenuatum } \\
\text { Isaria farinosa }\end{array}$} & & & & $\operatorname{Inf}$ & Sup \\
\hline & $0 \mathrm{a}$ & $0 \mathrm{a}$ & $30.00 \pm 0.00 \mathrm{a}$ & 30.00 & 30.00 \\
\hline & $100 \mathrm{bc}$ & $100 \mathrm{~b}$ & $8.60 \pm 1.47 \mathrm{bc}$ & 5.51 & 11.48 \\
\hline & $40 \mathrm{ac}$ & $20 \mathrm{a}$ & $20.60 \pm 5.16 \mathrm{ac}$ & 10.47 & 30.72 \\
\hline & $20 \mathrm{a}$ & $0 \mathrm{a}$ & $29.60 \pm 0.35 a$ & 28.89 & 30.30 \\
\hline
\end{tabular}

a Tiempo medio de vida (AST) limitado a 30 días. Resultados en la misma columna con la misma letra no son significativamente diferentes $(\alpha=0.05)$.

${ }^{\mathrm{b}}$ Medias en la misma columna seguidas por la misma letra no son significativamente diferentes (test $\chi 2, \mathrm{P}<0.05$ ).

\subsection{Transmisión horizontal de B. pseudobassiana entre adultos de M. galloprovincialis}

\subsubsection{Utilizando suspensión conidial}

Los valores de AST de los insectos tratados fueron significativamente más bajos (24.80 días para los machos, 23.60 días para las hembras) y la mortalidad fue mayor que la de los controles (Tab. 3). Ninguno de los sexos inoculados transmitieron el hongo a sus parejas control. Sólo se observó crecimiento micelial en los insectos inoculados.

\subsubsection{Utilizando formulación seca}

Los valores de AST de los insectos tratados fueron significativamente más bajos 
(4.60 días para los machos, 5.00 días para las hembras) y la mortalidad fue mayor que la de los controles (Tab. 3). Se encontró clara evidencia de transmisión horizontal y ambos sexos inoculados transmitieron el hongo a sus parejas control.

Tabla 3. Transmisión de la cepa Beauveria pseudobassiana EABps 11/01-Mg de Monochamus galloprovincialis adultos inoculados a adultos control del sexo contrario.

\begin{tabular}{|c|c|c|c|c|c|c|c|}
\hline \multirow{3}{*}{ Ensayo $^{a}$} & \multirow{3}{*}{ Inoculado } & \multirow{3}{*}{ Control } & \multicolumn{5}{|c|}{ Formulación conidial seca } \\
\hline & & & \multicolumn{2}{|c|}{ Mortalidad (\%)c, d } & \multicolumn{3}{|c|}{ Análisis de supervivencia Kaplan-Meier } \\
\hline & & & Total & $\begin{array}{l}\text { Crecimiento } \\
\text { micelial }\end{array}$ & $\begin{array}{c}\text { ASTa } \\
(\text { media } \pm \text { SE })\end{array}$ & \multicolumn{2}{|c|}{$\begin{array}{c}\text { Intervalo de } \\
\text { confianza } 95 \%\end{array}$} \\
\hline \multirow{3}{*}{ I } & & \multirow{3}{*}{$\begin{array}{l}\text { q } \\
\text { to } \\
\text { On }\end{array}$} & \multirow[b]{2}{*}{$0 \mathrm{a}$} & \multirow[b]{2}{*}{ 0a } & \multirow[b]{2}{*}{$23.60 \pm 3.08 \mathrm{a}$} & $\operatorname{Inf}$ & Sup \\
\hline & & & & & & 17.53 & 29.66 \\
\hline & & & 0a & 0a & $24.80 \pm 2.94 \mathrm{a}$ & 19.02 & 30.57 \\
\hline II & $\sigma^{\lambda}$ & q & $\begin{array}{c}0 \mathrm{a}^{\prime} \\
100 \mathrm{~b}^{\prime}\end{array}$ & $\begin{array}{c}0 \mathrm{a}^{\prime} \\
57.14 \mathrm{~b}\end{array}$ & $\begin{array}{l}23.60 \pm 3.44 \mathrm{~A} \\
12.80 \pm 1.22 \mathrm{~B}\end{array}$ & $\begin{array}{l}16.84 \\
10.39\end{array}$ & $\begin{array}{l}30.35 \\
15.20\end{array}$ \\
\hline III & 우 & $\hat{o}$ & $\begin{array}{c}100 \mathrm{~A} \\
16.66 \mathrm{~B}\end{array}$ & $\begin{array}{c}42.86 \mathrm{~A} \\
0 \mathrm{~B}\end{array}$ & $\begin{array}{l}11.40 \pm 1.44 \mathrm{a}^{\prime} \\
27.20 \pm 1.58 \mathrm{~b}^{\prime}\end{array}$ & $\begin{array}{c}8.56 \\
24.09\end{array}$ & $\begin{array}{l}14.23 \\
30.30\end{array}$ \\
\hline IV & $\begin{array}{l}q \\
+ \\
0\end{array}$ & & $\begin{array}{l}100 \mathrm{~A}^{\prime} \\
83.33 \mathrm{~A}^{\prime}\end{array}$ & $\begin{array}{c}100 \mathrm{~A} \\
42.86 \mathrm{~A}\end{array}$ & $\begin{array}{l}10.00 \pm 0.60 \mathrm{~A}^{\prime} \\
13.80 \pm 2.07 \mathrm{~A}^{\prime}\end{array}$ & $\begin{array}{l}8.69 \\
9.73\end{array}$ & $\begin{array}{l}11.30 \\
17.86\end{array}$ \\
\hline \multirow{3}{*}{ Ensayo $^{a}$} & \multirow{3}{*}{ Inoculado } & \multirow{3}{*}{ Control } & \multicolumn{5}{|c|}{ Formulación conidial seca } \\
\hline & & & \multicolumn{2}{|c|}{ Mortalidad (\%)c, d } & \multicolumn{3}{|c|}{ Análisis de supervivencia Kaplan-Meier } \\
\hline & & & Total & $\begin{array}{l}\text { Crecimiento } \\
\text { micelial }\end{array}$ & $\begin{array}{c}\text { ASTa } \\
(\text { media } \pm \text { SE })\end{array}$ & $\begin{array}{r}\text { Inte } \\
\text { confi }\end{array}$ & $\begin{array}{l}\mathrm{de} \\
95 \%\end{array}$ \\
\hline \multirow{3}{*}{ I } & & \multirow{3}{*}{$\begin{array}{l}\text { Q } \\
\hat{0}\end{array}$} & & & & $\operatorname{Inf}$ & Sup \\
\hline & & & 0a & $0 \mathrm{a}$ & $25.00 \pm 2.46 \mathrm{a}$ & 20.17 & 29.82 \\
\hline & & & 0a & $0 \mathrm{a}$ & $21.10 \pm 3.71 \mathrm{a}$ & 13.81 & 28.38 \\
\hline II & $0^{\pi}$ & ㅇ & $\begin{array}{l}100 a^{\prime} \\
100 a^{\prime}\end{array}$ & $\begin{array}{c}\text { 0a' } \\
33.33 \mathrm{~b}\end{array}$ & $\begin{array}{c}10.00 \pm 2.47 \mathrm{~A} \\
5.00 \pm 0.00 \mathrm{~A}\end{array}$ & $\begin{array}{l}5.14 \\
5.00\end{array}$ & $\begin{array}{c}14.85 \\
5.00\end{array}$ \\
\hline III & 우 & \multirow[t]{2}{*}{$0^{\pi}$} & $\begin{array}{l}100 \mathrm{~A} \\
100 \mathrm{~A}\end{array}$ & $\begin{array}{l}40 \mathrm{~A} \\
0 \mathrm{~B}\end{array}$ & $\begin{array}{c}5.50 \pm 0.28 \mathrm{a}^{\prime} \\
10.40 \pm 2.22 \mathrm{~b}^{\prime}\end{array}$ & $\begin{array}{l}4.97 \\
6.04\end{array}$ & $\begin{array}{c}6.03 \\
14.75\end{array}$ \\
\hline IV & $\begin{array}{l}\text { q } \\
0 \\
0\end{array}$ & & $\begin{array}{l}100 A^{\prime} \\
100 A^{\prime}\end{array}$ & $\begin{array}{c}0 \mathrm{~A}^{\prime} \\
50 \mathrm{~B}^{\prime}\end{array}$ & $\begin{array}{l}5.00 \pm 0.36 \mathrm{~A}^{\prime} \\
4.60 \pm 0.45 \mathrm{~A}^{\prime}\end{array}$ & $\begin{array}{l}4.28 \\
3.71\end{array}$ & $\begin{array}{l}5.71 \\
5.48\end{array}$ \\
\hline
\end{tabular}

${ }^{a}$ Ensayo (I) machos control $\mathrm{x}$ hembras control, (II) machos inoculados $\mathrm{x}$ hembras control, (III) machos control $\mathrm{x}$ hembras inoculadas $\mathrm{y}$ (IV) machos inoculados $\mathrm{x}$ hembras inoculadas.

${ }^{\mathrm{b}}$ En el mismo ensayo, resultados en la misma columna con la misma letra no son significativamente diferentes $(\alpha=0.05)$. Tiempo medio de vida (AST) limitado a 30 días.

${ }^{\mathrm{c}}$ Medias en la misma columna seguidas por la misma letra no son significativamente diferentes (test $\chi 2, \mathrm{P}<0.05$ ).

${ }^{\mathrm{d}}$ Mortalidad corregida por Abbott. 


\subsection{Transmisión vertical de B. pseudobassiana entre adultos de $M$. galloprovincialis}

\subsubsection{Utilizando suspensión conidial}

No se encontró evidencia de transmisión vertical. Aunque las hembras control realizaron más mordeduras de puesta que las inoculadas (Tab. 4). Tampoco hubo diferencias en la progenie viva dos semanas después de la oviposición (Fig. 1A).
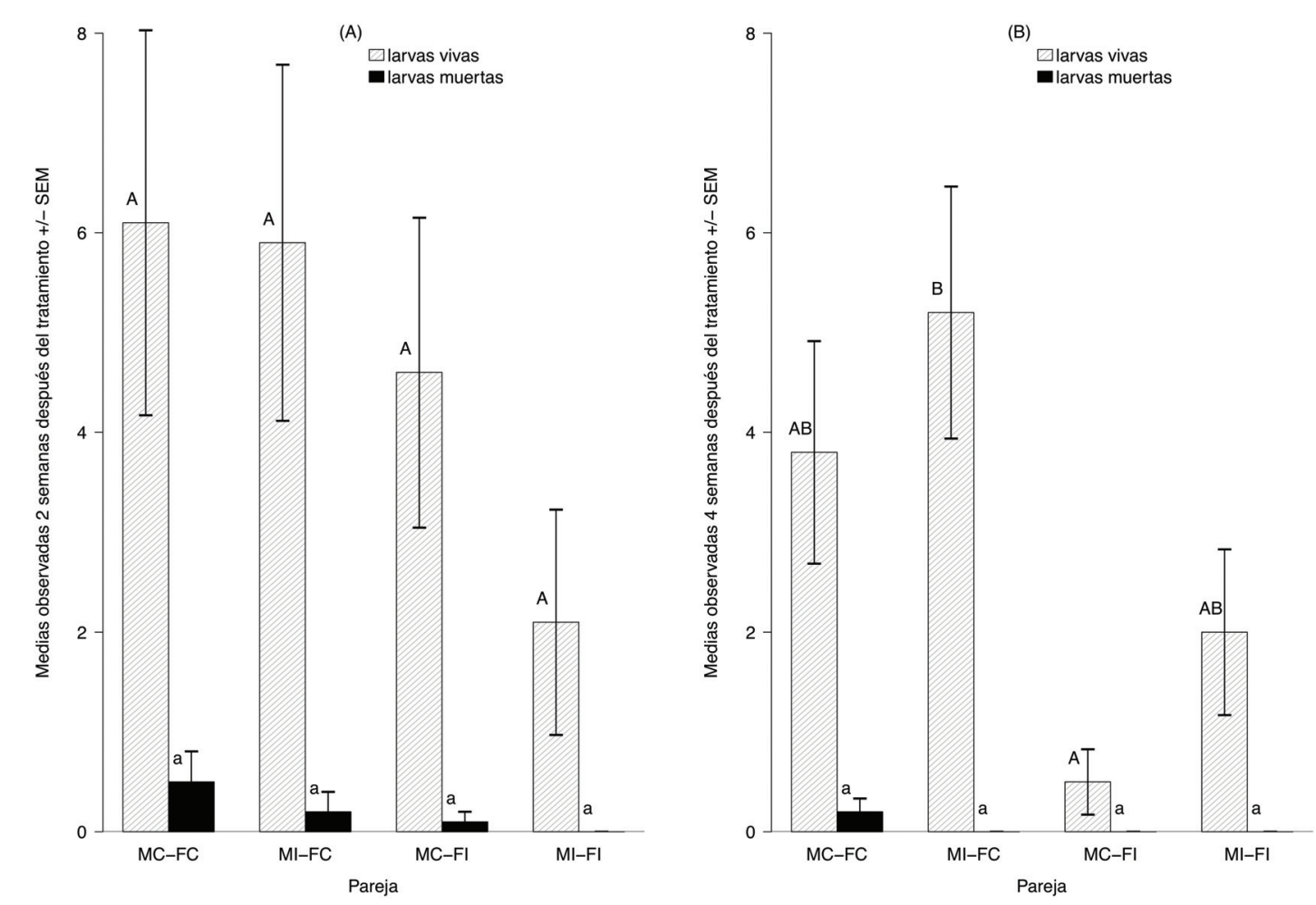

Figura 1. Número medio de larvas vivas y muertas dos (A) y cuatro (B) semanas después de la oviposición en el experimento de transmisión vertical con suspensión conidial. (MC: Macho control; MI: Macho infectado; FC: Hembra control; FI: Hembra infectada). Las barras para larvas vivas ( $\mathrm{F}=1.45, \mathrm{P}=0.244, \mathrm{df}=3$ ) y muertas $(\mathrm{F}=1.29, \mathrm{P}=0.292, \mathrm{df}=3)$ en $(\mathrm{A})$ y para larvas vivas $(\mathrm{F}=5.06, \mathrm{P}=0.005, \mathrm{df}=3)$ y muertas $(\mathrm{F}=2.09$, $\mathrm{P}=0.12, \mathrm{df}=3$ ) en $(\mathrm{B})$ seguidas de la misma letra no son significativamente diferentes (Test de diferencia significativa honesta de Tukey).

\subsubsection{Utilizando formulación seca}

Los resultados indican una reducción de la progenie inducida por transmisión horizontal y las parejas control produjeron significativamente más progenie que el resto (Tab. 4). El número de larvas vivas tras cinco días y el de larvas en el xilema a los seis meses fue significativamente superior en las hembras control que en el resto (Fig. 2). Las hembras control emparejadas con machos inoculados presentaron 
resultados similares a las hembras inoculadas, indicando que habían sido infectadas por sus parejas.

Tabla 4. Efecto de la cepa Beauveria pseudobassiana EABps 11/01-Mg en la progenie.

\begin{tabular}{|c|c|c|c|c|c|c|}
\hline \multirow[b]{2}{*}{ Ensayo $^{a}$} & \multirow[b]{2}{*}{ Inoculado } & \multirow[b]{2}{*}{ Control } & \multicolumn{2}{|c|}{ Suspensión conidial } & \multicolumn{2}{|c|}{ Formulación conidial seca } \\
\hline & & & $\begin{array}{l}\text { Mordeduras } \\
\text { de oviposición, }{ }^{\mathrm{d}} \\
(\text { media } \pm \text { SE) }\end{array}$ & $\begin{array}{c}\text { huevos, }{ }^{\mathrm{d}} \\
(\text { media } \pm \text { SE) }\end{array}$ & $\begin{array}{l}\text { Mordeduras } \\
\text { de oviposición, }{ }^{\mathrm{d}} \\
(\text { media } \pm \text { SE) }\end{array}$ & $\begin{array}{c}\text { huevos, }{ }^{\mathrm{d}} \\
(\text { media } \pm \mathrm{SE})\end{array}$ \\
\hline I & & $\begin{array}{l}\text { p } \\
\text { t }\end{array}$ & $16.35 \pm 2.07 \mathrm{a}$ & $5.40 \pm 1.15 \mathrm{ab}$ & $19.89 \pm 4.76 b$ & $11.22 \pm 2.85 b$ \\
\hline II & $\hat{O}$ & q & $17.75 \pm 1.92 \mathrm{a}$ & $5.80 \pm 1.04 \mathrm{a}$ & $3.22 \pm 1.44 \mathrm{a}$ & $0.89 \pm 0.61 \mathrm{a}$ \\
\hline III & q & $\sigma^{\lambda}$ & $7.15 \pm 1.67 \mathrm{~b}$ & $3.21 \pm 0.94 \mathrm{ab}$ & $1.60 \pm 0.76 \mathrm{a}$ & $0.10 \pm 0.10 \mathrm{a}$ \\
\hline IV & $\begin{array}{l}q \\
+ \\
0\end{array}$ & & $4.95 \pm 1.32 b$ & $2.05 \pm 0.68 \mathrm{~b}$ & $1.80 \pm 0.79 \mathrm{a}$ & $0.80 \pm 0.70 \mathrm{a}$ \\
\hline
\end{tabular}

${ }^{a}$ Ensayo (I) machos control $\mathrm{x}$ hembras control, (II) machos inoculados $\mathrm{x}$ hembras control, (III) machos control x hembras inoculadas y (IV) machos inoculados $\mathrm{x}$ hembras inoculadas.

${ }^{\mathrm{b}}$ Mordeduras realizadas por las hembras en la corteza para ovipositar, tanto con huevo como sin él.

${ }^{c}$ Huevos realmente puestos en las mordeduras de oviposición.

${ }^{d}$ Medias en la misma columna seguidas por la misma letra no son significativamente diferentes $(\alpha=0.05)$.

\section{Discusión}

Se han aislado por primera vez los hongos entomopatógenos Beauveria pseudobassiana, Isaria farinosa y Lecanicillium attenuatum de adultos y pupas de M. galloprovincialis. En Asia se había aislado B. bassiana y B. brongniartii de Monochamus sp. (Shin et al. 2009, 2011) de los cuales el primero se ha estudiado como agente control de vectores de B. xylophilus en Japón, Corea e Italia (Francardi et al. 2003; Shimazu, 1994; Shimazu et al. 1995). B. pseudobassiana EABps 11/01-Mg ha demostrado ser el mejor aislado contra $M$. galloprovincialis causando un $100 \%$ de mortalidad. Se han encontrado resultados similares para larvas y adultos de $\mathrm{Mo}$ nochamus saltuarius y Mechotypa diphysis tratados con Beauveria bassiana a $1 \cdot 10^{8}$ conidios/ml (Shin et al. 2009).

Se sabe que el nematodo es inoculado en árboles sanos por ambos sexos durante la alimentación (Mamiya and Enda, 1972) o en árboles moribundos por las hembras al ovipositar (Evans et al., 2008). La eficacia del hongo en un área infectada dependería de su habilidad para matar a los insectos antes de que se produzca el pico máximo de transmisión a los árboles sanos (Shimazu, 2004b). En laboratorio se ha demostrado que esta ventana temporal abarca las semanas 2 a 6 tras la emergencia de $M$. galloprovincialis (Naves et al., 2007), similar a M. alternatus en Japón (Togashi, 1985). 


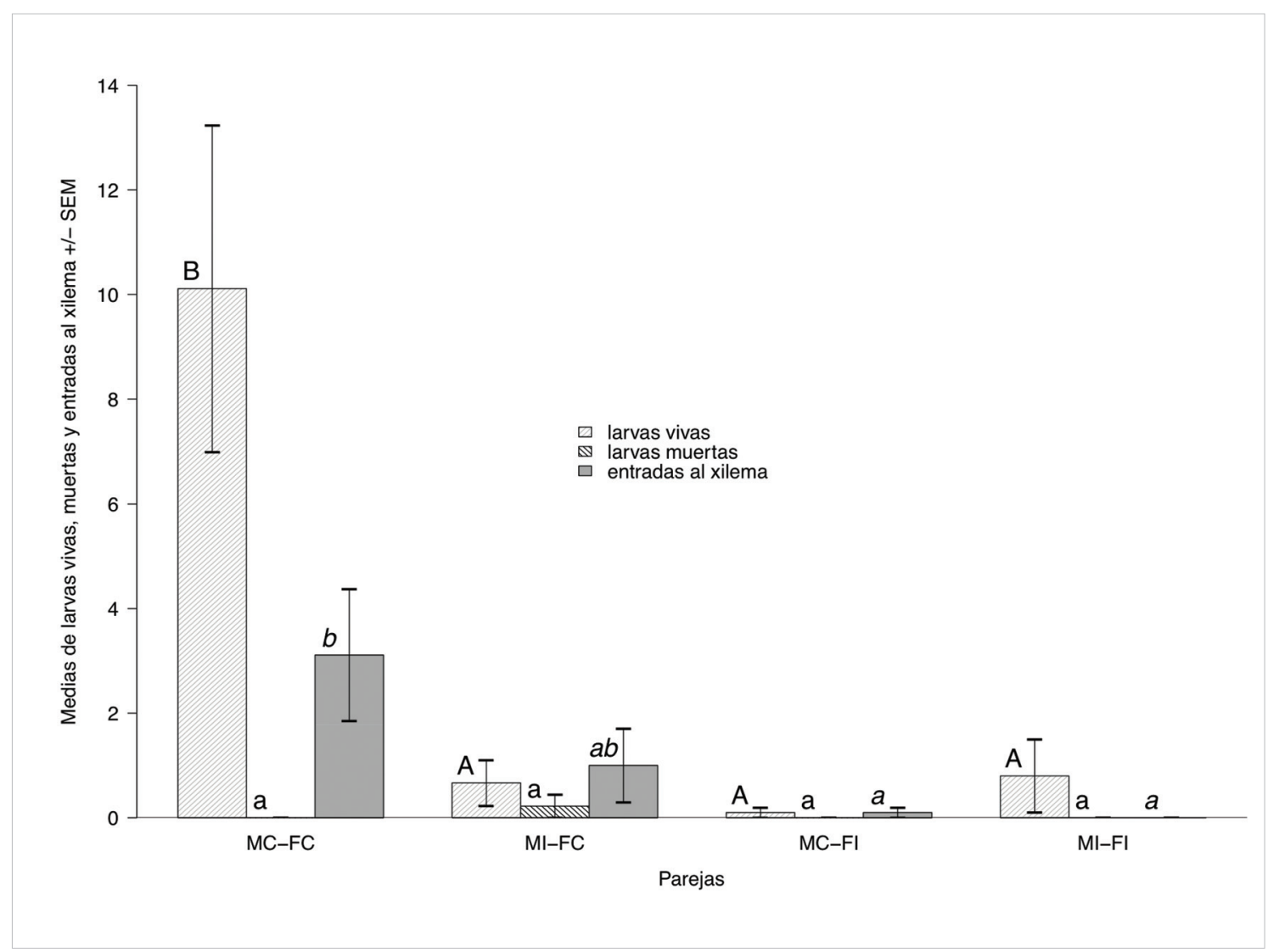

Figura 2. Número medio de larvas vivas y muertas y de entradas de larvas al xilema para el experimento de transmisión vertical con formulación seca. (MC: Macho control; MI: Macho infectado; FC: Hembra control; FI: Hembra infectada). Las barras para larvas vivas $(\mathrm{F}=14.00, \mathrm{P}<0.001, \mathrm{df}=3)$ y muertas $(\mathrm{F}=1.08$, $\mathrm{P}<0.37, \mathrm{df}=3)$ y para entradas al xilema $(\mathrm{F}=8.16, \mathrm{P}<0.001, \mathrm{df}=3)$ seguidas de la misma letra no son significativamente diferentes (Test de diferencia significativa honesta de Tukey).

Hemos observado que los adultos de M. galloprovincialis inoculados con la cepa B. pseudobassiana EABps 11/01-Mg en formulación seca la han transmitido a otros insectos del sexo contrario. Ya se sabía que las formulaciones secas de hongos entomopatógenos eran más adecuadas para estrategias de autodiseminación que las formulaciones líquidas (Quesada-Moraga et al. 2008). En nuestro estudio sólo se ha encontrado evidencia de transmisión horizontal con la formulación seca, lo que indica que el tipo de formulación influye en la transmisión. Puesto que muchos conidios pueden perderse en el ambiente, es posible que a través de los dispositivos de autodiseminación los insectos pueden dispersar conidios que podrían infectar a otros ejemplares independientemente de su sexo. Nuestros ensayos han revelado además una reducción significativa de la progenie, no como resultado de un aumento en la mortalidad de huevos o larvas sino como una reducción de su número puestos por hembras infectadas a través de machos contaminados, demostrando una reducción de progenie inducida por transmisión horizontal. Hamilton and Schal (1990) observaron que hembras de Blatella germanica superviventes a la exposición a un insecticida pusieron un menor número de ootecas. Quesada-Moraga et al. (2004) encon- 
tró resultados similares en la misma especie cuando fue inoculada con Metarihizium anisopliae.

Las estrategias de autodiseminación pueden facilitar la aplicación práctica de hongos entomopatógenos en áreas forestales y las tácticas de "atrae e infecta" se ajustan bien a este objetivo (Francardi et al., 2013; Klein and Lacey, 1999; Yasuda, 1999). Actualmente existe un sistema de trampeo eficaz para M. galloprovincialis mediante cebos (Pajares et al., 2010) y trampas (Álvarez et al., 2014). Aunque los cebos para este insecto son eficaces sólo sobre ejemplares adultos, éstos se alimentan sobre ramillos durante toda su vida (2-3 meses) por lo que la transmisión de nematodos a árboles sanos no sólo ocurriría durante la alimentación de maduración sino durante un periodo más extenso. Los insectos podrían ser atraídos a trampas específicamente preparadas para inocularles con la cepa $B$. pseudobassiana EABps $11 / 01-\mathrm{Mg}$, permitiéndoles después diseminarla por ellos mismos matando insectos durante la mayor parte de su periodo de infección de nematodos.

Concluimos que B. pseudobassiana podría ser utilizado en el manejo integrado de los vectores del nematodo de la madera del pino; que este estudio es el primer registro de aislamiento de B. pseudobassiana en M. galloprovincialis; que este aislado causa una gran mortalidad en los adultos de esta especie cuando es aplicado en forma de preparación conidial seca y que su autodiseminación por medio de técnicas de "atrae e infecta" puede suponer una nueva herramienta prometedora para el manejo de la enfermedad del marchitamiento de los pinos en áreas de alto riesgo.

\section{Agradecimientos}

Agradecemos a J.C. Domínguez, A. Martín, G. Pérez, P. Zamora y V. Rodríguez (Centro de Sanidad Forestal de Calabazanos, Junta de Castilla y León) su asistencia técnica. Este estudio ha sido financiado por el Ministerio de Economía y Competitividad (RTA2011-00069-C03-03).

\section{Bibliografía}

Abbott, W.S., 1925. A method of computing the effectiveness of an insecticide. J. Econ. Entomol. 18, 265-267

Álvarez, G., Etxebeste, I., Gallego, D., David, G., Bonifacio, L., Jactel, H., Sousa, E., Pajares, J.A., 2014. Optimization of traps for live trapping of Pine Wood Nematode vector Monochamus galloprovincialis. J. Appl. Entomol. n/a-n/a. doi:10.1111/jen.12186

Barnett, H.L., Hunter, B.B., 2006. Illustrated genera of imperfect fungi. APS Press, St. Paul, MN.

Crawley, M.J., 2007. The R book. John Wiley \& Sons, U.K.

Daub, M., 2008. Investigations on pathogenicity, invasion biology and population dynamics of the pine wood nematode Bursaphelenchus xylophilus (Steiner und Buhrer 1934) Nickle 1970 in European conifers. Julius Kühn-Institut, Braunschweig. 
Espárrago, G., 2012. Resultados de trampeos en Sierra de Dios Padre 2011. Informe I e Informe II. Junta de Extremadura. Presented at the VII Taller de Feromonas de Escolítidos.

Evans, S., Evans, H., Ikegami, M., 2008. Modeling PWN-Induced Wilt Expression: A Mechanistic Approach, in: Pine Wilt Disease: A worldwide threat to forest ecosystems. Springer, Dordrecht, pp. 259-278.

Finney, D.J., 1971. Probit analysis. Cambridge University Press, Cambridge, United Kingdom.

Francardi, V., Benvenuti, C., Barzanti, G.P., Roversi, P.F., 2013. Autocontamination trap with entomopathogenic fungi: a possible strategy in the control of Rhynchophorus ferrugineus (Olivier) (Coleoptera Curculionidae). Redia-G. Zool. 96, 57-67.

Francardi, V., Ruminc P, deSilva J, 2003. On microbial control of Monochamus galloprovincialis (Olivier) (Coleoptera Cerambycidae) by means of Beauveria bassiana (Bals.) Vuillemin (Deuterornycotina Hyphomycetes). Redia 86, 129-132.

Garrido-Jurado, I., Marquez, M., Ortiz-Urquiza, A., Santiago-Alvarez, C., Iturriaga, EA., Quesada-Moraga, E., Monte, E., Hermosa, R., 2011. Genetic analyses place most Spanish isolates of Beauveria bassiana in a molecular group with world-wide distribution. BMC Microbiol. 11, 84.

Hamilton, R.L., Schal, C., 1990. Sublethal effects of chlorpyrifos-methyl on reproduction in female German cockroaches (Dictyoptera, Blattellidae). J. Econ. Entomol. 83, 441-443.

Ibeas, F., Diez, J.J., Pajares, J.A., 2008. Olfactory sex attraction and mating behaviour in the pine sawyer Monochamus galloprovincialis (Coleoptera: Cerambycidae). J. Insect Behav. $21,101-110$.

Ibeas, F., Gallego, D., Diez, J.J., Pajares, J.A., 2007. An operative kairomonal lure for managing pine sawyer beetle Monochamus galloprovincialis (Coleoptera: Cerymbycidae). $J$. Appl. Entomol. 131, 13-20. doi:10.1111/j.1439-0418.2006.01087.x

Kamata, N., 2008. Integrated Pest Management of Pine Wilt Disease in Japan: Tactics and strategies, in: Zhao, B., Futai, K., Sutherland, J., Takeuchi, Y. (Eds.), Pine Wilt Disease. Springer Japan, pp. 304-322.

Kaplan, E., Meier, P., 1958. Nonparametric-Estimation from incomplete observations. J. Am. Stat. Assoc. 53, 457-481. doi:10.2307/2281868

Kishi, Y., 1995. The pine wood nematode and the Japanese pine sawyer. Thomas, Tokyo.

Klein, M.G., Lacey, L.A., 1999. An attractant trap for autodissemination of entomopathogenic fungi into populations of the Japanese beetle Popillia japonica (Coleoptera: Scarabaeidae). Biocontrol Sci. Technol. 9, 151-158.

Linit, M.J., 1988. Nematode vector relationships in the pine wilt disease system. J. Nematol. 20, 227-235.

Linit, M.J., Akbulut, S., 2008. The insect vectors: Biology and ecology, in: Pine Wilt Disease: A worldwide threat to forest ecosystems. Springer, pp. 211-213.

Mamiya, Y., Enda, N., 1972. Transmission of Bursaphelenchus lignicolus (Nematoda-Aphelenchoididae) by Monochamus alternatus (Coleoptera-Cerambycidae). Nematologica $18,159-162$.

Mota, M.M., Braasch, H., Bravo, M.A., Penas, A.C., Burgermeister, W., Metge, K., Sousa, E., 1999. First report of Bursaphelenchus xylophilus in Portugal and in Europe. Nematology 1, 727-734. doi:10.1163/156854199508757

Naves, P., 2008. Biology of Monochamus galloprovincialis (Coleoptera, Cerambycidae) in the pine wilt disease affected zone, Southern Portugal. Silva Lusit. 16, 133-148. 
Naves, P.M., Camacho, S., De Sousa, E.M., Quartau, J.A., 2007. Transmission of the pine wood nematode Bursaphelenchus xylophilus through feeding activity of Monochamus galloprovincialis (Col., Cerambycidae). J. Appl. Entomol. 131, 21-25. doi:10.1111/ j.1439-0418.2006.01111.x

O'Donnell, K., Cigelnik, E., Nirenberg, H.I., 1998. Molecular systematics and phylogeography of the Gibberella fujikuroi species complex. Mycologia 90, 465-493.

Okitsu M, 2000. Control of adults of Monochamus alternatus Hope (Coleoptera: Cerambycidae) by application of non-woven fabric strips containing Beauveria bassiana (Deuteromycotina: Hyphomycetes) on infested tree trunks. J. Jpn. Soc. 82, 276-280.

Pajares, J.A., 2010. Identification and field activity of a male-produced aggregation pheromone in the pine sawyer beetle, Monochamus galloprovincialis. J. Chem. Ecol. 36, 14.

Pajares, J.A., Ibeas, F., Diez, J.J., Gallego, D., 2004. Attractive responses by Monochamus galloprovincialis (Col., Cerambycidae) to host and bark beetle semiochemicals. J. Appl. Entomol. 128, 633-638. doi:10.1111/j.1439-0418.2004.00899.633-638

Phan, L.K., 2008. Potential of entomopathogenic nematodes for controlling the Japanese pine sawyer, Monochamus alternatus, in: Zhao, B., Futai, K., Sutherland, J., Takeuchi, Y., Phan, L. (Eds.), Pine Wilt Disease. Springer Japan, pp. 371-379.

Quesada-Moraga, E., Santos-Quirós, R., Valverde-García, P., Santiago-Álvarez, C., 2004. Virulence, horizontal transmission, and sublethal reproductive effects of Metahizium anisopliae (Anamorphic fungi) on the German cochroach (Blattodea: Blattellidae). J. Invertebr. Pathol. 87, 51-58.

Quesada-Moraga, E., Martin-Carballo, I., Garrido-Jurado, I., Santiago-Álvarez, S., 2008. Horizontal transmission of Metarhizium anisopliae among laboratory populations of Ceratitis capitata (Wiedemann) (Diptera: Tephritidae). Biol. Control. 47, 115-124.

$\mathrm{R}$ Core Team, 2012. R: A language and environment for statistical computing. R Foundation for Statistical Computing, Vienna, Austria.

Raeder, U., Broda, P., 1985. Rapid preparation of DNA from filamentous fungi. Lett. Appl. Microbiol. 1, 17-20.

Rodrigues, J.M., 2008. National eradication programme for the pinewood nematode, in: Pine Wilt Disease: A worldwide threat to forest ecosystems, Ed by Mota, M., Vieira, P., Springer Netherlands, pp. 5-14.

Shimazu, M., 1994. Potential of the cerambycid-parasitic type of Beauveria brongniartii (Deuteromycotina, Hyphomycetes) for microbial control of Monochamus alternatus Hope (Coleoptera, Cerambycidae). Appl. Entomol. Zool. 29, 127-130.

Shimazu, M., 2004a. A novel technique to inoculate conidia of entomopathogenic fungi and its application for investigation of susceptibility of the Japanese pine sawyer, Monochamus alternatus, to Beauveria bassiana. Appl. Entomol. Zool. 39, 485-490. doi:10. 1303/aez.2004.485

Shimazu, M., 2004b. Effects of temperature on growth of Beauveria bassiana F-263, a strain highly virulent to the Japanese pine sawyer, Monochamus alternatus, especially tolerance to high temperatures. Appl. Entomol. Zool. 39, 469-475. doi:10.1303/aez.2004.469

Shimazu, M., 2008. Biological Control of the Japanese pine sawyer beetle, Monochamus alternatus, in: Zhao, B., Futai, K., Sutherland, J., Takeuchi, Y., Shimazu, M. (Eds.), Pine Wilt Disease. Springer Japan, pp. 351-370.

Shimazu, M., Sato, H., 2003. Effects of larval age on mortality of Monochamus alternatus Hope (Coleoptera: Cerambycidae) after application of nonwoven fabric strips with Beau- 
veria bassiana. Appl. Entomol. Zool. 38, 1-5.

Shimazu, M., Tsuchiya, D., Sato, H., Kushida, T., 1995. Microbial control of Monochamus alternatus hope (Coleoptera, Cerambycidae) by application of nonwoven fabric strips with Beauveria bassiana (Deuteromycotina, Hyphomycetes) on Infested Tree Trunks. Appl. Entomol. Zool. 30, 207-213.

Shin, T.Y., Choi, J.B., Bae, S.M., Cha, Y.R., Oh, J.M., Koo, H.N., 2009. Isolation and identification of entomopathogenic fungus from the pine wilt disease vector, Monochamus alternatus Hope (Coleoptera: Cerambycidae) in Korea. Int. J. Indust. Entomol. 18, 125- 129.

Shin, S., 2008. Pine wilt disease in Korea, in: Pine wilt disease, Ed. by Zhao, B.G., Futai, K., Sutherland, J.R., Takeuchi, Y., Springer, Japan, 26-32.

Shin, T.Y., Choi, J.B., Bae, S.M., Koo, H.N., Roh, J.Y., Je, Y.H., Jin, B.R., Woo, S.D., 2011. Characterization of Beauveria bassiana MsW1 isolated from pine sawyers, Monochamus saltuarius. J. Basic. Microb. 51, 531-539.

Sousa, E., Bravo, M.A., Pires, J., Naves, P., Penas, A.C., Bonifacio, L., Mota, M.M., 2001. Bursaphelenchus xylophilus (Nematoda; Aphelenchoididae) associated with Monochamus galloprovincialis (Coleoptera; Cerambycidae) in Portugal. Nematology 3, 89-91. doi:10.1163/156854101300106937

Throne, J.E., Weaver, D. K., Chew, V., Baker, J.E., 1995. Probit anaysis of correlated data: multiple observations over time at one concentration. J. Econ. Entomol. 88, 1510-1512.

Togashi, K., 1985. Transmission curves of Bursaphelenchus xylophilus (Nematoda, Aphelenchoididae) from its vector, Monochamus alternatus (Coleoptera, Cerambycidae), to pine trees with reference to population performance. Appl. Entomol. Zool. 20, 246-251.

Tomiczek, C. and Hoyer-Tomiczek, U., 2008. Biology studies relevant to the vector role of Monochamus Species for pine wood nematode, in: Mota, M. and Vieira, P. (Eds), Pine Wilt Disease: A worldwide threat to forest ecosystems. Springer Netherlands, pp. 215-220.

Vives, E., 2000. Coleoptera, Cerambycidae, in: Ramos, M.A. (Ed.), Fauna Ibérica. Museo Nacional de Ciencias Naturales, CSIC, Madrid.

Wingfield, M.J., 1982. Association of pine wood nematode with stressed trees in Minnesota, Iowa and Wisconsin. Plant Dis. 934-937.

$\mathrm{Xu}, \mathrm{F} ., 2008$. Recent advances in the integrated management of the pine wood nematode in China, in: Zhao, B., Futai, K., Sutherland, J., Takeuchi, Y. (Eds.), Pine Wilt Disease. Springer Japan, pp. 323-333.

Yasuda, K., 1999. Auto-infection system for the sweet potato weevil, Cylas formicarius (Fabricius) (Coleoptera: Curculionidae) with entomopathogenic fungi, Beauveria bassiana using a modified sex pheromone trap in the field. Appl. Entomol. Zool. 34, 501-505.

Zhao, B., 2008. Pine Wilt Disease in China, in: Pine wilt disease, Ed. by Zhao, B.G., Futai, K., Sutherland, J.R., Takeuchi, Y., Springer, Japan, 18-25. 
